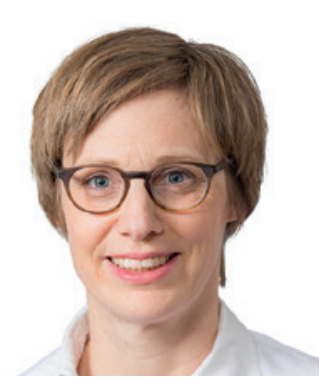

\title{
Orbitale Traumata
}

\author{
Ameli Gabel-Pfisterer
}

Augenklinik, Klinikum Ernst-von-Bergmann, Potsdam, Deutschland

Zusammenfassung zu Zorn M, et al.: Trauma der Orbita und Hinweise zur Radiologischen Diagnostik. Augenheilkunde up2date 2021; 11:1-16.

\section{Zusammenfassung}

Orbitale Traumata können sich vielfältig präsentieren und durch eine Vielzahl beteiligter neurovaskulärer Strukturen im Knochenkäfig eine visusbedrohende Verletzung darstellen. Eine adäquate radiologische Darstellung ist wichtig, um eine unter Umständen zeitkritische und eventuell interdisziplinäre Therapie mit HNO, MKG oder Neurochirurgie zügig einleiten zu können.

(c) 2021 Gabel-Pfistere 


\section{Transfer in die Praxis}

\section{Hintergrund}

Bei bis zu einem Viertel aller Gesichtsverletzungen ist die Orbita beteiligt.

Neben einer direkten Verletzung des Augapfels und des Sehnerven bergen Orbitaverletzungen ein Risiko für alle durch diesen starren, knöchern begrenzten Raum ziehenden neurovaskulären Strukturen. Insbesondere eine Kompression des Nervus opticus und der Blutgefäße, die die ischämiesensible Netzhaut versorgen, stellen ein Risiko für eine akute oder dauerhafte Visuseinschränkung dar. Bei der initialen Untersuchung ist ein Verständnis der Anatomie und der möglichen Traumamechanismen für die Einleitung einer effizienten, richtungsweisenden Diagnostik und nachfolgenden Therapie für den Funktionserhalt des Auges von entscheidender Bedeutung. Kenntnisse über die Möglichkeiten radiologischer Darstellung verschiedener Pathologien sind hilfreich, um auch in akuten Situationen zu einem schnellen und präzisen Befund als Grundlage für eine adäquate Therapie zu gelangen.

In der gut bebilderten Übersichtsarbeit beschreiben wir die aktuelle Literatur bezüglich Diagnostik und Therapie von Orbitafrakturen, intraorbitalen Fremdkörperverletzungen, des orbitalen Kompartmentsyndroms und traumatischer Optikusneuropathien und geben Hinweise zur radiologischen Darstellung der Pathologien.

\section{Fazit für die Praxis}

Um einen Hinweis auf den möglichen Verletzungsmechanismus zu erhalten, ist eine zielgerichtete Anamnese mit Fragen zum Unfallhergang und zur Akutsymptomatik, aber auch zu Vorerkrankungen und einer eventuellen Antikoagulation von Bedeutung.

Die klinische Untersuchung wird unter Umständen durch Komorbiditäten eingeschränkt, dennoch wird empfohlen neben der visuellen Funktion besonders auf die Symmetrie der Bulbusstellung, die Motilität und Blutungen zu achten, die Hinweis auf eine Bulbusverletzung sein können. Auch die Prüfung eines afferenten Pupillendefizits (rAPD) ist mit geringem technischen Aufwand möglich. Eine Spaltlampenuntersuchung und Funduskopie zur Evaluation von Bulbusverletzungen sollte zusätzlich erfolgen.

\section{Orbitafrakturen}

Blowout-Traumata der Orbita entstehen nach Kraftübertragung durch den tonisierten Bulbus auf die umgebenden Knochen [1] und betreffen meist den Orbitaboden, während eine direkte Stauchung eines Orbitawandknochens zu einer Fraktur des fragilsten Knochens des Orbitarahmens führt [2], was meist die mediale Lamina papyracea ist.

Der Orbitaboden stellt, in 25-40\% der Fälle nach einem Schlag von vorne, die häufigste Lokalisation [3, 4] einer Fraktur dar, die durch Einklemmung des Musculus rectus inferior zu Motilitätseinschränkung und Muskeldefekten, aber auch zu vegetativen Reaktionen führen kann. In Kombination mit einer Fraktur der medialen Orbitawand kann ein Enophthalmus auftreten [3, 5]. Mediale Orbitawandfrakturen sind Folge eines Mittelgesichtstraumas und können neben Muskeleinklemmungen durch Epistaxis, Liquorrhoe und bei Beteiligung der Arteria ethmoidalis anterior durch ein intraorbitales Hämatom gekennzeichnet sein. Laterale Orbitawand- und Orbitadachfrakturen sind seltener als die vorgenannten, da sie erst bei größerer Krafteinwirkung auftreten, können aber ebenso zu Motilitätseinschränkungen und Parästhesien durch Nerveneinklemmung oder Schwellung des orbitalen Gewebes führen. Da durch den Apex der Orbita eine Vielzahl kritischer neurovaskulärer Leitungsbahnen führt, muss dieser Bereich in der klinischen und radiologischen Diagnostik von Orbitafrakturen ebenfalls betrachtet werden.

Zur radiologischen Primärdiagnostik und Operationsplanung wird zunächst eine hochauflösende Dünnschicht-CT empfohlen, die im Verdachtsfall von Gefäß-, Muskel- oder Sehnervenverletzung oder -Kompression und nach Ausschluss eines eventuellen Metallfremdkörpers durch eine MRT-Untersuchung ergänzt werden sollte. Die Dringlichkeit einer operativen Intervention wird nach AWMF-Leitlinien bei persistierendem okulokardialem Reflex als «soforts und bei eingeklemmten Weichteilen als «dringend klassifiziert, während persistierende Bulbusfehlstellung oder Diplopie ohne Muskeleinklemmungen auch im späteren Verlauf operativ versorgt werden können $[6,7]$.

\section{Intraorbitale Fremdkörper und orbitales Kompartmentsyndrom}

Intraorbitale Fremdkörper, besonders nach der typischen HammerMeisselverletzung [8], sind oft metallisch und können radiologisch im CT lokalisiert werden. Ein CT trägt außerdem zur orientierenden Materialbestimmung bei. Organische Fremdkörper hingegen können ein zusätzliches MRT erforderlich machen und das Risiko von Infektionen mit sich bringen. Bei Fremdkörpern der anterioren Orbita kann eine Sonographie, etwa eine Hochfrequenz-Sonographie $(50 \mathrm{mHz})$ eine Lokalisation möglich machen.

Durch Blutungen oder Infektionen kann in der räumlich limitierten Orbita ein Kompartmentsyndrom entstehen, das zu einer Kompression neurovaskulärer Strukturen mit Visusverlust und Ophthalmoplegie führen kann. Ein Funktionsverlust ist zu befürchten, wenn der intraorbitale Druck den retinalen Perfusionsdruck übersteigt. Die kurze Ischämietoleranz insbesondere der Netzhaut macht diese Situation zu einem Notfall $[9,10]$. Bei Visusminderung, rAPD oder einem intraokularen Druck von mehr als $40 \mathrm{mmHg}$ ist eine unmittelbare operative Entlastung durch Kantotomie und Augendrucksenkung erforderlich. Die CT-Diagnostik kann zunächst als Spiral-CT nativ erfolgen und Informationen zum Verletzungsgrad liefern. Bei einer Schichtdicke von 1-2 mm und koronarer und sagittaler MPR im Knochen- und im Weichteilfenster ist es möglich eine hämorrhagische, ödematöse oder emphysematische Genese zu differenzieren und die intra-, extrakonale oder apikale Lokalisation zu erkennen. 


\section{Traumatische Optikusneuropathie (TON)}

Nach komplexen Gesichtsschädelverletzungen und seltener nach Schädelhirntraumen kann es durch Fremdkörper oder Knochenfragmente zu einer direkten Schädigung des Nervus opticus kommen [11]. Eine operative Intervention zur Entlastung, Fremdkörperentfernung oder Rekonstruktion knöcherner Strukturen kann daher kurzfristig notwendig werden. Eine indirekte TON wird als Spätfolge einer mechanischen Belastung des Sehnerven und der Ganglienzellen beschrieben [5] und kann sich auch zeitverzögert als Visusverlust oder Gesichtsfelddefekt manifestieren und ist therapeutisch oft nicht zu beeinflussen.

\section{Disclosure Statement}

No financial disclosures.

\section{Literatur}

1 Smith B, Regan WF Jr: Blow-out fracture of the orbit; mechanism and correction of internal orbital fracture. Am J Ophthalmol. 1957:44:733-739.

2 Fujino T, Makino K: Entrapment mechanism and ocular injury in orbital blowout fracture. Plast Reconstr Surg. 1980;65:571-574.

3 Iftikhar M, Canner JK, Hall L, et al.: Characteristics of orbital floor fractures in the United States from 2006 to 2017. Ophthalmology. 2021;128:463-470.
4 Ko MJ, Morris CK, Kim JW, et al.: Orbital fractures: national inpatient trends and complications. Ophthalmic Plast Reconstr Surg. 2013;29:298-303.

5 Kersten RC, Vagefi MR, Bartley GB: Orbital "blowout" fractures: time for a new paradigm. Ophthalmology. 2018;125:796-798.

6 Burnstine MA: Clinical recommendations for repair of isolated orbital floor fractures: an evidence-based analysis. Ophthalmology. 2002;109: 1207-1210; discussion 1210-1211; quiz 1212-1213.

7 Deutsche Gesellschaft für Mund-, Kiefer- und Gesichtschirurgie e.V. (DGMKG): S2e-Leitlinie Rekonstruktion von Orbitadefekten. AWMF-Registernr. 007/099; 2013. Im Internet (Stand: 24.08.2021): www.awmf.org/uploads/ tx_szleitlinien/007_099|_S2e_Rekonstruktion_Orbita_2013-07_01.pdf

8 Fulcher TP, McNab AA, Sullivan TJ: Clinical features and management of intraorbital foreign bodies. Ophthalmology. 2002;109:494-500.

9 Lima V, Burt B, Leibovitch I, et al.: orbital compartment syndrome: the ophthalmic surgical emergency. Surv Ophthalmol. 2009;54:441-449.

10 Kopecky A, Rokohl AC, Nemcansky J, et al.: Das retrobulbäre Hämatom eine potenziell visusbedrohende Komplikation. Klin Monbl Augenheilkd. 2019;238:609-615.

11 Jamal BT, Pfahler SM, Lane KA, et al.: Ophthalmic injuries in patients with zygomaticomaxillary complex fractures requiring surgical repair. J Oral Maxillofac Surg. 2009;67:986-989.

Korrespondenz an:

Ameli Gabel-Pfisterer, agabel-pfisterer @ klinikumevb.de 